\title{
Retrieve the Bethe states of quantum integrable models solved via the off-diagonal Bethe Ansatz
}

\section{Xin Zhang ${ }^{a}$, Yuan-Yuan $\mathbf{L i}^{a}$, Junpeng Cao ${ }^{a, b}$, Wen-Li Yang ${ }^{c, d 1}$, Kangjie $\mathrm{Shi}^{c}$ and Yupeng Wang, ${ }^{a} 2$}

${ }^{a}$ Beijing National Laboratory for Condensed Matter Physics, Institute of Physics, Chinese Academy of Sciences, Beijing 100190, China

${ }^{b}$ Collaborative Innovation Center of Quantum Matter, Beijing, China

${ }^{c}$ Institute of Modern Physics, Northwest University, Xian 710069, China

${ }^{d}$ Beijing Center for Mathematics and Information Interdisciplinary Sciences, Beijing, 100048, China

\begin{abstract}
Based on the inhomogeneous $T-Q$ relation constructed via the off-diagonal Bethe Ansatz, a systematic method for retrieving the Bethe-type eigenstates of integrable models without obvious reference state is developed by employing certain orthogonal basis of the Hilbert space. With the XXZ spin torus model and the open XXX spin$\frac{1}{2}$ chain as examples, we show that for a given inhomogeneous $T-Q$ relation and the associated Bethe Ansatz equations, the constructed Bethe-type eigenstate has a well-defined homogeneous limit.
\end{abstract}

PACS: 75.10.Pq, 03.65.Vf, 71.10.Pm

Keywords: Spin chain; Bethe Ansatz; $T-Q$ relation; Scalar product

\footnotetext{
${ }^{1}$ Corresponding author: wlyang@nwu.edu.cn

${ }^{2}$ Corresponding author: yupeng@iphy.ac.cn
} 


\section{Introduction}

The algebraic Bethe Ansatz method provides a powerful tool to solve integrable models with $U(1)$ symmetry [1, 2, 3, 4]. In that approach, both eigenvalues and eigenstates of transfer matrix can be constructed simultaneously. However, for integrable models without $U(1)$ symmetry, Bethe-type eigenstates can be constructed only for some very special boundary conditions [5, 6, 7, 8, 9, 10, 11, 12, 13]. Recently, a new method, namely, the off-diagonal Bethe Ansatz (ODBA) method [14, 15, 16, 17, 18] was proposed to approach exact solutions of generic integrable models either with or without $U(1)$ symmetry. In such an approach, the spectrum of the transfer matrix as well as the scalar products between off-shell states and an eigenstate can be observed without using any information of states. The central point of this method lies in construction of the inhomogeneous $T-Q$ relation based on operator product identities. An interesting issue left in this framework is how to retrieve eigenstates from the obtained spectrum. In principle, if the eigenvalues of a matrix are known, its eigenvectors should be determined completely. A remarkable progress in this aspect is the conjecture on the Bethe states of the open $X X X$ spin chain [19] based on the inhomogeneous $T-Q$ relation. On the other hand, the eigenstates of several integrable models in case of inhomogeneity were also derived via the separation of variables (SoV) method [20, 21, 22]. However, how to reach the homogeneous limit of the SoV states and how to prove the conjecture proposed in [19] still remain open.

In this paper, we propose a systematic method to retrieve the eigenstates from the ODBA solutions. The central point lies in that from the inhomogeneous $T-Q$ relation, one can retrieve the reference state that is normally not known. With this reference state, one can easily reach the homogeneous limit of the Bethe states. We employ two archetype integrable models without $U(1)$ symmetry, i.e., the $X X Z$ spin torus model and the open $X X X$ spin- $\frac{1}{2}$ chain with generic boundary fields as examples, to elucidate our method.

The paper is organized as follows. Section 2 serves as an introduction of our notations and brief review of the ODBA solutions of the inhomogeneous spin torus. In section 3, after introducing a complete (both left and right) basis of the Hilbert space, we retrieve the Bethe-type eigenstates of the transfer matrix with the help of the inhomogeneous $T-Q$

relations. Section 4 is devoted to constructing Bethe states of the open $X X X$ spin- $\frac{1}{2}$ chain with generic boundary fields. In section 5 , we summarize our results and give the concluding 
remarks. Some technical proofs are given in Appendices A and B respectively.

\section{ODBA Solution of the Spin Torus}

The $X X Z$ spin torus model is described by the Hamiltonian

$$
H=-\sum_{n=1}^{N}\left(\sigma_{n}^{x} \sigma_{n+1}^{x}+\sigma_{n}^{y} \sigma_{n+1}^{y}+\cosh \eta \sigma_{n}^{z} \sigma_{n+1}^{z}\right),
$$

with the anti-periodic boundary condition

$$
\sigma_{N+1}^{x}=\sigma_{1}^{x}, \quad \sigma_{N+1}^{y}=-\sigma_{1}^{y}, \quad \sigma_{N+1}^{z}=-\sigma_{1}^{z}
$$

The integrability of this model, associated with the following $\bar{R}$-matrix satisfying the quantum Yang-Baxter equation (QYBE), has been studied by several authors [23, 24, 25, 26, 27]

$$
\bar{R}(u)=\frac{1}{\sinh \eta}\left(\begin{array}{llll}
\sinh (u+\eta) & & & \\
& \sinh u & \sinh \eta & \\
& \sinh \eta & \sinh u & \\
& & & \sinh (u+\eta)
\end{array}\right)
$$

where the generic complex number $\eta$ is the crossing parameter.

Let us introduce the "row-to-row" (or one-row ) monodromy matrix $T_{0}(u)$, a $2 \times 2$ matrix with operator-valued elements acting on $\mathbf{V}^{\otimes N}$,

$$
\begin{aligned}
T_{0}(u) & =\bar{R}_{0 N}\left(u-\theta_{N}\right) \bar{R}_{0 N-1}\left(u-\theta_{N-1}\right) \cdots \bar{R}_{01}\left(u-\theta_{1}\right) \\
& =\left(\begin{array}{cc}
\bar{A}(u) & \bar{B}(u) \\
\bar{C}(u) & \bar{D}(u)
\end{array}\right) .
\end{aligned}
$$

Here $\left\{\theta_{j} \mid j=1, \cdots, N\right\}$ are arbitrary free complex parameters which are usually called inhomogeneous parameters. QYBE implies that the monodromy matrix given in (2.4) satisfies the following RTT relation

$$
\bar{R}_{12}(u-v) T_{1}(u) T_{2}(v)=T_{2}(v) T_{1}(u) \bar{R}_{12}(u-v)
$$

Moreover, the corresponding transfer matrix $t(u)$ is given by

$$
t(u)=\operatorname{tr}_{0}\left(\sigma_{0}^{x} T_{0}(u)\right)=\bar{B}(u)+\bar{C}(u)
$$

The QYBE and the $Z_{2}$-symmetry of the $R$-matrix lead to the fact that the transfer matrices with different spectral parameters commute with each other: $[t(u), t(v)]=0$. 
The Hamiltonian (2.1) with anti-periodic boundary condition is then expressed in terms of the transfer matrix by

$$
H=-2 \sinh \eta\left\{\left.\frac{\partial \ln t(u)}{\partial u}\right|_{u=0,\left\{\theta_{j}=0\right\}}-\frac{1}{2} N \operatorname{coth} \eta\right\}
$$

It was shown [14] that an eigenvalue $\Lambda(u)$ of $t(u)$ satisfies the following properties:

$$
\begin{aligned}
& \Lambda(u+i \pi)=(-1)^{N-1} \Lambda(u), \\
& \Lambda(u), \text { as function of } u, \text { is a trigonometrical polynomial of degree } N-1, \\
& \Lambda\left(\theta_{j}\right) \Lambda\left(\theta_{j}-\eta\right)=-\bar{a}\left(\theta_{j}\right) \bar{d}\left(\theta_{j}-\eta\right), \quad j=1, \cdots, N .
\end{aligned}
$$

Here $\bar{a}(u)=\prod_{l=1}^{N} \sinh \left(u-\theta_{l}+\eta\right)$ and $\bar{d}(u)=\bar{a}(u-\eta)=\prod_{l=1}^{N} \sinh \left(u-\theta_{l}\right)$. The above relations completely determine the eigenvalue $\Lambda(u)$ as follows [14]: the eigenvalue $\Lambda(u)$ is given by the following inhomogeneous $T-Q$ relation

$$
\Lambda(u)=e^{u} \bar{a}(u) \frac{Q_{1}(u-\eta)}{Q_{2}(u)}-e^{-u-\eta} \bar{d}(u) \frac{Q_{2}(u+\eta)}{Q_{1}(u)}-c(u) \frac{\bar{a}(u) \bar{d}(u)}{Q_{1}(u) Q_{2}(u)}
$$

where

$$
Q_{1}(u)=\prod_{j=1}^{M} \frac{\sinh \left(u-\mu_{j}\right)}{\sinh \eta}, \quad Q_{2}(u)=\prod_{j=1}^{M} \frac{\sinh \left(u-\nu_{j}\right)}{\sinh \eta}
$$

and $c(u)$ is an adjust function. For an even $N$ and $M=\frac{N}{2}, c(u)$ is given by

$$
c(u)=e^{u+\sum_{l=1}^{N} \theta_{l}-M \eta-2 \sum_{j=1}^{M} \mu_{j}}-e^{-u-\eta-\sum_{l=1}^{N} \theta_{l}+M \eta+2 \sum_{j=1}^{M} \nu_{j}},
$$

where the $N$ parameters $\left\{\mu_{j}\right\}$ and $\left\{\nu_{j}\right\}$ satisfy the following Bethe Ansatz equations (BAEs)

$$
\begin{aligned}
& \bar{d}\left(\nu_{j}\right)=\frac{e^{\nu_{j}}}{c\left(\nu_{j}\right)} Q_{1}\left(\nu_{j}-\eta\right) Q_{1}\left(\nu_{j}\right), \quad j=1, \cdots, M, \\
& \bar{a}\left(\mu_{j}\right)=-\frac{e^{-\mu_{j}-\eta}}{c\left(\mu_{j}\right)} Q_{2}\left(\mu_{j}+\eta\right) Q_{2}\left(\mu_{j}\right), \quad j=1, \cdots, M .
\end{aligned}
$$

For an odd $N$ and $M=\frac{N+1}{2}, c(u)$ is given by

$$
c(u)=\frac{1}{2 \sinh \eta}\left\{e^{2 u+\sum_{l=1}^{N} \theta_{l}-M \eta-2 \sum_{j=1}^{M} \mu_{j}}+e^{-2 u-2 \eta-\sum_{l=1}^{N} \theta_{l}+M \eta+2 \sum_{j=1}^{M} \nu_{j}}\right\},
$$

and the $N+1$ parameters $\left\{\mu_{j}\right\}$ and $\left\{\nu_{j}\right\}$ satisfy the BAEs (2.14)-(2.15) with the adjust function $c(u)$ given by (2.16) . 
It was known in [15] that there actually exist many different types of $T-Q$ relations for the solutions to (2.8)-(2.10) and each of them gives the complete set of eigenvalues of the transfer matrix as proven in [28]. Here we present another simple $T-Q$ relation for $\Lambda(u)$, which corresponds to the $M=0$ type in [15], namely,

$$
\Lambda(u)=\bar{a}(u) e^{u} \frac{Q(u-\eta)}{Q(u)}-e^{-u-\eta} \bar{d}(u) \frac{Q(u+\eta)}{Q(u)}-c(u) \frac{\bar{a}(u) \bar{d}(u)}{Q(u)}
$$

with

$$
Q(u)=\prod_{j=1}^{N} \frac{\sinh \left(u-\lambda_{j}\right)}{\sinh \eta}
$$

and

$$
c(u)=e^{u-N \eta+\sum_{j=1}^{N}\left(\theta_{j}-\lambda_{j}\right)}-e^{-u-\eta-\sum_{j=1}^{N}\left(\theta_{j}-\lambda_{j}\right)} .
$$

The $N$ parameters $\left\{\lambda_{j}\right\}$ in (2.18) satisfy the associated BAEs

$$
e^{\lambda_{j}} \bar{a}\left(\lambda_{j}\right) Q\left(\lambda_{j}-\eta\right)-\bar{d}\left(\lambda_{j}\right) e^{-\lambda_{j}-\eta} Q\left(\lambda_{j}+\eta\right)-c\left(\lambda_{j}\right) \bar{a}\left(\lambda_{j}\right) \bar{d}\left(\lambda_{j}\right)=0, \quad j=1, \cdots, N
$$

\section{$3 \quad$ Retrieving the Eigenstates}

With generic inhomogeneous parameters $\theta_{j} \neq \theta_{l}$ and $\theta_{j} \neq \theta_{l}-\eta$, a set of orthogonal states

parameterized by the $N$ inhomogeneous constants $\left\{\theta_{j} \mid j=1, \cdots, N\right\}$ that form a basis of the Hilbert space exists. In the framework of ODBA, such a basis is quite useful to prove the retrieved Bethe states to be eigenstates of the transfer matrix.

\subsection{Orthogonal basis}

The RTT relation (2.5) of the monodromy matrix $T(u)$ given by (2.4) gives rise to some quadratic commutation relations among its matrix elements. Here we present some relevant ones for our purpose

$$
\begin{aligned}
& {[\bar{B}(u), \bar{B}(v)]=[\bar{C}(u), \bar{C}(v)]=0,} \\
& \bar{A}(u) \bar{B}(v)=\frac{\sinh (u-v-\eta)}{\sinh (u-v)} \bar{B}(v) \bar{A}(u)+\frac{\sinh \eta}{\sinh (u-v)} \bar{B}(u) \bar{A}(v), \\
& \bar{D}(u) \bar{B}(v)=\frac{\sinh (u-v+\eta)}{\sinh (u-v)} \bar{B}(v) \bar{D}(u)-\frac{\sinh \eta}{\sinh (u-v)} \bar{B}(u) \bar{D}(v),
\end{aligned}
$$




$$
\begin{aligned}
& \bar{C}(u) \bar{A}(v)=\frac{\sinh (u-v+\eta)}{\sinh (u-v)} \bar{A}(v) \bar{C}(u)-\frac{\sinh \eta}{\sinh (u-v)} \bar{A}(u) \bar{C}(v), \\
& \bar{C}(u) \bar{D}(v)=\frac{\sinh (u-v-\eta)}{\sinh (u-v)} \bar{D}(v) \bar{C}(u)+\frac{\sinh \eta}{\sinh (u-v)} \bar{D}(u) \bar{C}(v), \\
& {[\bar{C}(u), \bar{B}(v)]=\frac{\sinh \eta}{\sinh (u-v)}[\bar{D}(u) \bar{A}(v)-\bar{D}(v) \bar{A}(u)] .}
\end{aligned}
$$

Let us introduce the all spin up state $|0\rangle$ and its dual state $\langle 0|$

$$
|0\rangle=\otimes_{j=1}^{N}|\uparrow\rangle_{j}, \quad\langle 0|=\left\langle\left.\uparrow\right|_{j} \otimes_{j=1}^{N},\right.
$$

which are nothing but the reference state and its dual in the framework of the algebraic Bethe Ansatz method [4. The elements of the monodromy matrix act on them as follows:

$$
\begin{aligned}
& \bar{A}(u)|0\rangle=\bar{a}(u)|0\rangle, \quad \bar{D}(u)|0\rangle=\bar{d}(u)|0\rangle, \quad \bar{C}(u)|0\rangle=0, \\
& \langle 0| \bar{A}(u)=\bar{a}(u)\langle 0|, \quad\langle 0| \bar{D}(u)=\bar{d}(u)\langle 0|, \quad\langle 0| \bar{B}(u)=0 .
\end{aligned}
$$

Let us introduce some left and right states parameterized by the $N$ inhomogeneous parameters $\left\{\theta_{j}\right\}$ as follows 3 :

$$
\begin{aligned}
& \left\langle\theta_{p_{1}}, \cdots, \theta_{p_{n}}\right|=\langle 0| \prod_{j=1}^{n} \bar{C}\left(\theta_{p_{j}}\right), \quad 1 \leq p_{1}<p_{2}<\cdots<p_{n} \leq N, \\
& \left|\theta_{q_{1}}, \cdots, \theta_{q_{n}}\right\rangle=\prod_{j=1}^{n} \bar{B}\left(\theta_{q_{j}}\right)|0\rangle, \quad 1 \leq q_{1}<q_{2}<\cdots<q_{n} \leq N .
\end{aligned}
$$

Due to the fact that $\bar{d}\left(\theta_{j}\right)=0$, with the help of (3.3) and (3.5) one may derive that these states are in fact the eigenstates of $\bar{D}(u)$

$$
\begin{aligned}
& \bar{D}(u)\left|\theta_{p_{1}}, \cdots, \theta_{p_{n}}\right\rangle=\bar{d}(u) \prod_{j=1}^{n} \frac{\sinh \left(u-\theta_{p_{j}}+\eta\right)}{\sinh \left(u-\theta_{p_{j}}\right)}\left|\theta_{p_{1}}, \cdots, \theta_{p_{n}}\right\rangle, \\
& \left\langle\theta_{p_{1}}, \cdots, \theta_{p_{n}}\right| \bar{D}(u)=\bar{d}(u) \prod_{j=1}^{n} \frac{\sinh \left(u-\theta_{p_{j}}+\eta\right)}{\sinh \left(u-\theta_{p_{j}}\right)}\left\langle\theta_{p_{1}}, \cdots, \theta_{p_{n}}\right| .
\end{aligned}
$$

Note that the total number of the right (or left) states given in (3.11) (or (3.10) is

$$
\sum_{n=0}^{N} \frac{N !}{(N-n) ! n !}=2^{N}
$$

\footnotetext{
${ }^{3}$ These states were used in [20] to construct Sklyanin's quantum separation of variables (SoV) [29] representations of the Yang-Baxter algebra associated with the trigonometric six-vertex $R$-matrix.
} 
Using the commutation relations (3.1)-(3.6), one may derive the following orthogonal relations between the left states and the right states

$$
\left\langle\theta_{p_{1}}, \cdots, \theta_{p_{n}} \mid \theta_{q_{1}}, \cdots, \theta_{q_{m}}\right\rangle=f_{n}\left(\theta_{p_{1}}, \cdots, \theta_{p_{n}}\right) \delta_{m, n} \prod_{j=1}^{n} \delta_{p_{j}, q_{j}}
$$

where

$$
f_{n}\left(\theta_{p_{1}}, \cdots, \theta_{p_{n}}\right)=\prod_{l=1}^{n}\left\{\bar{a}\left(\theta_{p_{l}}\right) \bar{d}_{p_{l}}\left(\theta_{p_{l}}\right) \prod_{k \neq l}^{n} \frac{\sinh \left(\theta_{p_{l}}-\theta_{p_{k}}+\eta\right)}{\sinh \left(\theta_{p_{l}}-\theta_{p_{k}}\right)}\right\}
$$

We remark that $f_{0}=\langle 0 \mid 0\rangle=1$. The function $\bar{d}_{l}(u)$ is defined as

$$
\bar{d}_{l}(u)=\prod_{j \neq l}^{N} \frac{\sinh \left(u-\theta_{j}\right)}{\sinh \eta}, \quad l=1, \cdots, N
$$

Thus for generic values $\left\{\theta_{j}\right\}$, these right (or left) states form an orthogonal right (or left) basis of the Hilbert space, and any right (or left) state can be decomposed as a unique linear combination of these basis.

\subsection{Retrieving the Bethe states}

Due to the fact that the left states $\left\{\left\langle\theta_{p_{1}}, \cdots, \theta_{p_{n}}|| n=0, \cdots, N, \quad 1 \leq p_{1}<p_{2}<\cdots<p_{n} \leq\right.\right.$ $N\}$ given by (3.10) form a basis of the dual Hilbert space, an eigenstate $|\Psi\rangle$ of the transfer matrix (2.6) is completely determined (up to an overall scalar factor) by the following set of scalar products

$$
F_{n}\left(\theta_{p_{1}}, \cdots, \theta_{p_{n}}\right)=\left\langle\theta_{p_{1}}, \cdots, \theta_{p_{n}} \mid \Psi\right\rangle, \quad n=0, \cdots, N
$$

with the first one $F_{0}=1$. It was shown in [14] that these products $F_{n}\left(\theta_{p_{1}}, \cdots, \theta_{p_{n}}\right)$ are given by

$$
F_{n}\left(\theta_{p_{1}}, \cdots, \theta_{p_{n}}\right)=\prod_{l=1}^{n} \Lambda\left(\theta_{p_{l}}\right), \quad n=1, \cdots, N .
$$

Let us consider the following Bethe state

$$
\left|\lambda_{1}, \cdots, \lambda_{N}\right\rangle=\prod_{j=1}^{N} \frac{\bar{D}\left(\lambda_{j}\right)}{\bar{d}\left(\lambda_{j}\right)}\left|\Omega ;\left\{\theta_{j}\right\}\right\rangle,
$$


where the parameters $\left\{\lambda_{j} \mid j=1, \cdots, N\right\}$ satisfy the BAEs (2.20) and $\left|\Omega ;\left\{\theta_{j}\right\}\right\rangle$ is a generalized reference state to be determined so that the scalar products between the Bethe state (3.19) and the basis (3.10) satisfy the conditions (3.18), namely,

$$
\left\langle\theta_{p_{1}}, \cdots, \theta_{p_{n}} \mid \lambda_{1}, \cdots, \lambda_{N}\right\rangle=\prod_{l=1}^{n} \Lambda\left(\theta_{p_{l}}\right), \quad n=1, \cdots, N
$$

For an eigenvalue $\Lambda(u)$ given by the $T-Q$ relation (2.17), its value at the inhomogeneous point $\theta_{j}$ takes a simple form:

$$
\Lambda\left(\theta_{j}\right)=\bar{a}\left(\theta_{j}\right) e^{\theta_{j}} \frac{Q\left(\theta_{j}-\eta\right)}{Q\left(\theta_{j}\right)}, \quad j=1, \cdots, N .
$$

With the help of the exchange relation (3.5) and the above relations, the conditions (3.20) are then equivalent to the following requirements on the reference state:

$$
\left\langle\theta_{p_{1}}, \cdots, \theta_{p_{n}} \mid \Omega ;\left\{\theta_{j}\right\}\right\rangle=\prod_{l=1}^{n} a\left(\theta_{p_{l}}\right) e^{\theta_{p_{l}}}, \quad n=0, \cdots, N, 1 \leq p_{1}<p_{2}<\cdots<p_{n} \leq N .
$$

It is remarked that the above conditions do not depend on the parameters $\left\{\lambda_{j} \mid j=1, \cdots, N\right\}$ which should satisfy the BAEs (2.20). Hence the relations (3.22) uniquely determine the reference state $\left|\Omega ;\left\{\theta_{j}\right\}\right\rangle$ up to a scalar factor, which actually does not depend upon the parameters $\left\{\lambda_{j} \mid j=1, \cdots, N\right\}$.

Let us propose the following Ansatz for the reference state $\left|\Omega ;\left\{\theta_{j}\right\}\right\rangle$ :

$$
\left|\Omega ;\left\{\theta_{j}\right\}\right\rangle=\sum_{l=0}^{\infty} \frac{\left(\tilde{B}^{-}\right)^{l}}{[l]_{q} !}|0\rangle=\sum_{l=0}^{N} \frac{\left(\tilde{B}^{-}\right)^{l}}{[l]_{q} !}|0\rangle
$$

where the $q$-integers $\left\{[l]_{q} \mid l=0, \cdots\right\}$ and the operator $\tilde{B}^{-}$are given by

$$
\begin{aligned}
& {[l]_{q}=\frac{1-q^{2 l}}{1-q^{2}}, \quad[0]_{q}=1,} \\
& {[l]_{q} !=[l]_{q}[l-1]_{q} \cdots[1]_{q}, \quad q=e^{\eta},} \\
& \tilde{B}^{-}=\lim _{u \rightarrow+\infty}\left\{\left(2 \sinh \eta e^{-u}\right)^{N-1} e^{\sum_{l=1}^{N} \theta_{l}} \bar{B}(u)\right\} .
\end{aligned}
$$

The definitions (2.3) and (2.4) allow us to obtain the explicit expression of the operator $\tilde{B}^{-}$ as

$$
\tilde{B}^{-}=\sum_{l=1}^{N} e^{\theta_{l}+\frac{(N-1) \eta}{2}} e^{\frac{\eta}{2} \sum_{k=l+1}^{N} \sigma_{k}^{z}} \sigma_{l}^{-} e^{-\frac{\eta}{2} \sum_{k=1}^{l-1} \sigma_{k}^{z}}
$$


Direct calculation shows that the state $\left|\Omega ;\left\{\theta_{j}\right\}\right\rangle$ given by (3.23) indeed satisfies the relations (3.22). The proof is given in Appendix A. Then we conclude that the Bethe state (3.19) with the corresponding reference state (3.23) is an eigenstate of the transfer matrix (2.6), provided that the parameters $\left\{\lambda_{j} \mid j=1, \cdots, N\right\}$ satisfy the associated BAEs (2.20). The corresponding eigenvalue is given by the $T-Q$ relation (2.17).

From the definitions (2.3) and (2.4), one can see that the operators $\bar{D}(u)$ and $\tilde{B}^{-}$have well-defined homogeneous limit when $\left\{\theta_{j} \rightarrow 0\right\}$. In the homogeneous limit, the reference state (3.23) becomes

$$
|\Omega\rangle=\lim _{\left\{\theta_{j} \rightarrow 0\right\}}\left|\Omega ;\left\{\theta_{j}\right\}\right\rangle=\sum_{l=0}^{\infty} \frac{\left(B^{-}\right)^{l}}{[l]_{q} !}|0\rangle=\sum_{l=0}^{N} \frac{\left(B^{-}\right)^{l}}{[l]_{q} !}|0\rangle,
$$

where the operator $B^{-}$(c.f., (3.27)) reads

$$
B^{-}=\lim _{\left\{\theta_{j} \rightarrow 0\right\}} \tilde{B}^{-}=\sum_{l=1}^{N} e^{\frac{(N-1) \eta}{2}} e^{\frac{\eta}{2} \sum_{k=l+1}^{N} \sigma_{k}^{z}} \sigma_{l}^{-} e^{-\frac{\eta}{2} \sum_{k=1}^{l-1} \sigma_{k}^{z}} .
$$

This implies that the homogeneous limit of the Bethe state (3.19) gives rise to the eigenstate 4 of the corresponding homogeneous transfer matrix. The corresponding eigenvalue and BAEs are given by the homogeneous limits of (2.17) and (2.20) respectively. It should be noted that in contrast to that used in the algebraic Bethe Ansatz scheme, the reference state (3.23) (or (3.28)) is no longer a pure product state but a highly entangled state (actually a $q$-spin coherent state).

Associated with the $T-Q$ relation (2.11), we can construct another type of Bethe states

$$
\left|\mu_{1}, \cdots, \mu_{M} ; \nu_{1}, \cdots, \nu_{M}\right\rangle=\prod_{j=1}^{M} \frac{\bar{D}\left(\mu_{j}\right)}{\bar{d}\left(\mu_{j}\right)} \frac{\bar{D}\left(\nu_{j}\right)}{\bar{d}\left(\nu_{j}\right)}\left|\bar{\Omega} ;\left\{\theta_{j}\right\}\right\rangle
$$

where the associated reference state is

$$
\left|\bar{\Omega} ;\left\{\theta_{j}\right\}\right\rangle=\sum_{n=0}^{N} \sum_{p} f_{n}^{-1}\left(\theta_{p_{1}}, \cdots, \theta_{p_{n}}\right) \prod_{l=1}^{n} e^{\theta_{p_{l}}} \bar{a}\left(\theta_{p_{l}}\right) \frac{Q_{1}\left(\theta_{p_{l}}\right)}{Q_{2}\left(\theta_{p_{l}}-\eta\right)}\left|\theta_{p_{1}}, \cdots, \theta_{p_{n}}\right\rangle,
$$

with the $Q$-functions $Q_{1}(u)$ and $Q_{2}(u)$ given by (2.12). It can be easily checked that

$$
\begin{aligned}
\left\langle\theta_{p_{1}}, \cdots, \theta_{p_{n}} \mid \mu_{1}, \cdots, \mu_{M} ; \nu_{1}, \cdots, \nu_{M}\right\rangle & =\prod_{l=1}^{n} e^{\theta_{p_{l}}} \bar{a}\left(\theta_{p_{l}}\right) \frac{Q_{1}\left(\theta_{p_{l}}-\eta\right)}{Q_{2}\left(\theta_{p_{l}}\right)} \\
& =\prod_{l=1}^{n} \Lambda\left(\theta_{p_{l}}\right)=F_{n}\left(\theta_{p_{1}}, \cdots, \theta_{p_{n}}\right) .
\end{aligned}
$$

\footnotetext{
${ }^{4}$ It is remarked that in contrast with the Hamiltonian given by (2.1) and (2.2), the reference state (3.28) is not invariant under the translation. This is due to that the reference state is no longer an eigenstate of the Hamiltonian.
} 
Therefore, the Bethe state (3.30) is also an eigenstate of the transfer matrix provided that the parameters $\left\{\mu_{j}\right\}$ and $\left\{\nu_{j}\right\}$ satisfy the associated BAEs (2.14)-(2.15). In the homogeneous limit, the reference state (3.31) reads

$$
|\bar{\Omega}\rangle=\lim _{\left\{\theta_{j} \rightarrow 0\right\}}\left|\bar{\Omega} ;\left\{\theta_{j}\right\}\right\rangle=\sum_{l=0}^{\infty} \frac{\left(Q_{1}(0) B^{-}\right)^{l}}{[l]_{q} !\left(Q_{2}(-\eta)\right)^{l}}|0\rangle=\sum_{l=0}^{N} \frac{\left(Q_{1}(0) B^{-}\right)^{l}}{[l]_{q} !\left(Q_{2}(-\eta)\right)^{l}}|0\rangle .
$$

Some remarks are in order. Different choice of the inhomogeneous $T-Q$ relations gives different parameterization of the eigenvalues and leads to a different expression of the Bethe state and the associated reference state 5 . The procedure for constructing the Bethe states (3.19) and (3.30) is different from that of the algebraic Bethe Ansatz. In the latter scheme, one uses known reference state and creation operator to derive eigenvalues and eigenstates of the transfer matrix, while in the ODBA scheme one uses known eigenvalues (in terms of inhomogeneous $T-Q$ relation) and creation operator to retrieve the reference state. The key point is that the eigenstates of the creation operator (i.e., $\bar{D}(u)$ ) form a basis of the Hilbert space. Such a reversed process makes it convenient to approach the eigenstate problem of quantum integrable models without obvious reference state.

\section{Results for the open XXX spin- $\frac{1}{2}$ chain}

In this section, we show how to retrieve the Bethe states conjectured in [19] from the ODBA solution of the open XXX spin- $\frac{1}{2}$ chain described by the Hamiltonian

$$
H=\sum_{j=1}^{N-1} \vec{\sigma}_{j} \cdot \vec{\sigma}_{j+1}+\frac{\eta}{p} \sigma_{1}^{z}+\frac{\eta}{q}\left(\xi \sigma_{N}^{x}+\sigma_{N}^{z}\right),
$$

where $p, q$ and $\xi$ are arbitrary boundary parameters. The corresponding transfer matrix of the inhomogeneous open chain is given by [3]

$$
t^{(o)}(u)=\operatorname{tr}_{0}\left(K_{0}^{+}(u) T_{0}(u) K_{0}^{-}(u) \hat{T}_{0}(u)\right),
$$

with the monodromy matrices $T(u)$ and $\hat{T}(u)$ defined as

$$
\begin{aligned}
& T_{0}(u)=R_{0 N}\left(u-\theta_{N}\right) \cdots R_{01}\left(u-\theta_{1}\right)=\left(\begin{array}{cc}
A(u) & B(u) \\
C(u) & D(u)
\end{array}\right), \\
& \hat{T}_{0}(u)=R_{01}\left(u+\theta_{1}\right) \cdots R_{0 N}\left(u+\theta_{N}\right)=(-1)^{N}\left(\begin{array}{cc}
D(-u-\eta) & -B(-u-\eta) \\
-C(-u-\eta) & A(-u-\eta)
\end{array}\right),
\end{aligned}
$$

\footnotetext{
${ }^{5}$ There exists a homogeneous $T-Q$ relation for $\Lambda(u)$ [24]. However, how to retrieve the corresponding Bethe state for such a parametrization is still an open and interesting problem.
} 
where the associated $R$-matrix $R(u)$ (c.f. $(2.3)$ ) reads

$$
R(u)=\left(\begin{array}{cccc}
u+\eta & & & \\
& u & \eta & \\
& \eta & u & \\
& & & u+\eta
\end{array}\right)
$$

and the $K$-matrices are given by [30, 31]

$$
\begin{aligned}
K^{-}(u) & =\left(\begin{array}{cc}
p+u & 0 \\
0 & p-u
\end{array}\right) \stackrel{\text { def }}{=}\left(\begin{array}{ll}
K_{11}^{-}(u) & K_{12}^{-}(u) \\
K_{21}^{-}(u) & K_{22}^{-}(u)
\end{array}\right), \\
K^{+}(u) & =\left(\begin{array}{cc}
q+u+\eta & \xi(u+\eta) \\
\xi(u+\eta) & q-u-\eta
\end{array}\right) \stackrel{\text { def }}{=}\left(\begin{array}{cc}
K_{11}^{+}(u) & K_{12}^{+}(u) \\
K_{21}^{+}(u) & K_{22}^{+}(u)
\end{array}\right) .
\end{aligned}
$$

The transfer matrix has the commutative property $[t(u), t(v)]=0$, and is therefore the generating functional of a family of commuting operators, among which is the Hamiltonian (4.1), i.e.,

$$
H=\left.\eta \frac{\partial \ln t^{(o)}(u)}{\partial u}\right|_{u=0, \theta_{j}=0}-N
$$

\subsection{ODBA solution and the associated basis}

Let us introduce the following functions

$$
a(u)=\prod_{l=1}^{N}\left(u-\theta_{l}+\eta\right), \quad d(u)=a(u-\eta)=\prod_{l=1}^{N}\left(u-\theta_{l}\right) .
$$

Each eigenvalue of the transfer matrix (4.2), denoted by $\Lambda^{(o)}(u)$, can be given in terms of the following inhomogeneous $T-Q$ relation [15, 32, 28] 6

$$
\begin{aligned}
\Lambda^{(o)}(u)= & (-1)^{N} \frac{2 u+2 \eta}{2 u+\eta}(u+p)\left(\sqrt{1+\xi^{2}} u+q\right) a(u) d(-u-\eta) \frac{Q(u-\eta)}{Q(u)} \\
& +(-1)^{N} \frac{2 u}{2 u+\eta}(u-p+\eta)\left(\sqrt{1+\xi^{2}}(u+\eta)-q\right) a(-u-\eta) d(u) \frac{Q(u+\eta)}{Q(u)} \\
& +2\left(1-\sqrt{1+\xi^{2}}\right) u(u+\eta) \frac{a(u) a(-u-\eta) d(u) d(-u-\eta)}{Q(u)},
\end{aligned}
$$

where the $Q$-function is given by

$$
Q(u)=\prod_{j=1}^{N}\left(u-\lambda_{j}\right)\left(u+\lambda_{j}+\eta\right) .
$$

\footnotetext{
${ }^{6}$ The $T-Q$ relation (4.9) corresponds to the special case (i.e., $M=0$ ) of the general ones proposed in [15]. A generalization to other cases is straightforward.
} 
The parameters $\left\{\lambda_{j}\right\}$ satisfy the following BAEs

$$
\begin{aligned}
1+ & \frac{\lambda_{j}\left(\lambda_{j}-p+\eta\right)\left(\sqrt{1+\xi^{2}}\left(\lambda_{j}+\eta\right)-q\right) a\left(-\lambda_{j}-\eta\right) d\left(\lambda_{j}\right) Q\left(\lambda_{j}+\eta\right)}{\left(\lambda_{j}+\eta\right)\left(\lambda_{j}+p\right)\left(\sqrt{1+\xi^{2}} \lambda_{j}+q\right) a\left(\lambda_{j}\right) d\left(-\lambda_{j}-\eta\right) Q\left(\lambda_{j}-\eta\right)} \\
= & (-1)^{N} \frac{\left(\sqrt{1+\xi^{2}}-1\right) \lambda_{j}\left(2 \lambda_{j}+\eta\right) a\left(-\lambda_{j}-\eta\right) d\left(\lambda_{j}\right)}{\left(\lambda_{j}+p\right)\left(\sqrt{1+\xi^{2}} \lambda_{j}+q\right) Q\left(\lambda_{j}-\eta\right)}, \quad j=1, \cdots, N .
\end{aligned}
$$

It is easy to check that the $K^{+}$-matrix can be diagonalized as

$$
\begin{aligned}
\bar{K}^{+}(u) & =U K^{+}(u) U^{-1}=\left(\begin{array}{cc}
q+\sqrt{1+\xi^{2}}(u+\eta) & 0 \\
0 & q-\sqrt{1+\xi^{2}}(u+\eta)
\end{array}\right) \\
& \stackrel{\text { def }}{=}\left(\begin{array}{cc}
\bar{K}_{11}^{+}(u) & 0 \\
0 & \bar{K}_{22}^{+}(u)
\end{array}\right),
\end{aligned}
$$

where the matrix $U$ is given by

$$
U=\left(\begin{array}{cc}
\xi & \sqrt{1+\xi^{2}}-1 \\
\xi & -\sqrt{1+\xi^{2}}-1
\end{array}\right)
$$

Accordingly, the gauged $K$-matrix $\bar{K}^{-}(u)$ reads

$$
\begin{aligned}
\bar{K}^{-}(u) & =U K^{-}(u) U^{-1}=\left(\begin{array}{cc}
p+\frac{1}{\sqrt{1+\xi^{2}}} u & \frac{\sqrt{1+\xi^{2}}-1}{\sqrt{1+\xi^{2}}} u \\
\frac{\sqrt{1+\xi^{2}}+1}{\sqrt{1+\xi^{2}}} u & p-\frac{1}{\sqrt{1+\xi^{2}}} u
\end{array}\right) \\
& \stackrel{\text { def }}{=}\left(\begin{array}{cc}
\bar{K}_{11}^{-}(u) & \bar{K}_{12}^{-}(u) \\
\bar{K}_{21}^{-}(u) & \bar{K}_{22}^{-}(u)
\end{array}\right) .
\end{aligned}
$$

Moreover, let us introduce two local states

$$
\begin{aligned}
|1\rangle_{n} & =\frac{\sqrt{1+\xi^{2}}+1}{2 \xi \sqrt{1+\xi^{2}}}|\uparrow\rangle_{n}+\frac{1}{2 \sqrt{1+\xi^{2}}}|\downarrow\rangle_{n}, \quad n=1, \cdots, N, \\
|2\rangle_{n} & =\frac{\sqrt{1+\xi^{2}}-1}{2 \xi \sqrt{1+\xi^{2}}}|\uparrow\rangle_{n}-\frac{1}{2 \sqrt{1+\xi^{2}}}|\downarrow\rangle_{n}, \quad n=1, \cdots, N,
\end{aligned}
$$

and their dual states

$$
\left\langle\left. 1\right|_{n}=\xi\left\langle\left.\uparrow\right|_{n}+\left(\sqrt{1+\xi^{2}}-1\right)\left\langle\left.\downarrow\right|_{n},\left\langle\left. 2\right|_{n}=\xi\left\langle\left.\uparrow\right|_{n}-\left(\sqrt{1+\xi^{2}}+1\right)\left\langle\left.\downarrow\right|_{n}, n=1, \cdots, N .\right.\right.\right.\right.\right.\right.
$$

These states satisfy the following orthogonal relations

$$
\left\langle\left. a\right|_{j} b\right\rangle_{k}=\delta_{a, b} \delta_{j, k}, \quad a, b=1,2, \quad j, k=1, \cdots, N .
$$


Based on the above local states, let us introduce two product states

$$
|\Omega\rangle_{\xi}=\otimes_{j=1}^{N}|1\rangle_{j}, \quad \xi\langle\bar{\Omega}|=\otimes_{j=1}^{N}\left\langle\left. 2\right|_{j} .\right.
$$

The double-row monodromy matrix of the present model reads

$$
\mathbb{T}(u)=T(u) K^{-}(u) \hat{T}(u)=\left(\begin{array}{ll}
\mathcal{A}(u) & \mathcal{B}(u) \\
\mathcal{C}(u) & \mathcal{D}(u)
\end{array}\right),
$$

and its gauged one is

$$
\begin{aligned}
\overline{\mathbb{T}}(u) & =U T(u) K^{-}(u) \hat{T}(u) U^{-1}=U T(u) U^{-1} U K^{-}(u) U^{-1} U \hat{T}(u) U^{-1} \\
& =\bar{T}(u) \bar{K}^{-}(u) \hat{\bar{T}}(u)=\left(\begin{array}{cc}
\overline{\mathcal{A}}(u) & \overline{\mathcal{B}}(u) \\
\overline{\mathcal{C}}(u) & \overline{\mathcal{D}}(u)
\end{array}\right) .
\end{aligned}
$$

The double-row monodromy matrix and its gauged one both satisfy the reflection algebra [3] and the exchange relations among $\mathcal{A}(u), \mathcal{B}(u), \mathcal{C}(u)$ and $\mathcal{D}(u)$ are the same as those among $\overline{\mathcal{A}}(u), \overline{\mathcal{B}}(u), \overline{\mathcal{C}}(u)$ and $\overline{\mathcal{D}}(u)$, which are listed in Appendix B. The transfer matrix $t^{(o)}(u)$ given by (4.2) can be expressed as

$$
\begin{aligned}
t^{(o)}(u) & =K_{11}^{+}(u) \mathcal{A}(u)+K_{12}^{+}(u) \mathcal{C}(u)+K_{21}^{+}(u) \mathcal{B}(u)+K_{22}^{+}(u) \mathcal{D}(u) \\
& =\bar{K}_{11}^{+}(u) \overline{\mathcal{A}}(u)+\bar{K}_{22}^{+}(u) \overline{\mathcal{D}}(u) .
\end{aligned}
$$

Noting that $\overline{\mathcal{C}}(u)$ forms a commuting family, i.e., $[\overline{\mathcal{C}}(u), \overline{\mathcal{C}}(v)]=0$, similarly as (3.10)(3.11) we can use its common (dual) eigenstates to construct the basis of right (left) Hilbert space. For this purpose, let us introduce the following right and left states parameterized by the $N$ inhomogeneous parameters $\left\{\theta_{j}\right\}$ :

$$
\begin{aligned}
\left.\left|\theta_{p_{1}}, \cdots, \theta_{p_{n}}\right\rangle\right\rangle & =\overline{\mathcal{A}}\left(\theta_{p_{1}}\right) \cdots \overline{\mathcal{A}}\left(\theta_{p_{n}}\right)|\Omega\rangle_{\xi}, \quad 1 \leq p_{1}<p_{2}<\cdots<p_{n} \leq N, \\
\left\langle\left\langle-\theta_{q_{1}}, \cdots,-\theta_{q_{n}}\right|\right. & =\xi\langle\bar{\Omega}| \overline{\mathcal{D}}\left(-\theta_{q_{1}}\right) \cdots \overline{\mathcal{D}}\left(-\theta_{q_{n}}\right), \quad 1 \leq q_{1}<q_{2}<\cdots<q_{n} \leq N .
\end{aligned}
$$

which are eigenstates of $\overline{\mathcal{C}}(u)$

$$
\begin{aligned}
\left.\overline{\mathcal{C}}(u)\left|\theta_{p_{1}}, \cdots, \theta_{p_{n}}\right\rangle\right\rangle & \left.=h\left(u,\left\{\theta_{p_{1}}, \cdots, \theta_{p_{n}}\right\}\right)\left|\theta_{p_{1}}, \cdots, \theta_{p_{n}}\right\rangle\right\rangle, \\
\left\langle\left\langle-\theta_{p_{1}}, \cdots,-\theta_{p_{n}}\right| \overline{\mathcal{C}}(u)\right. & =h^{\prime}\left(u,\left\{-\theta_{p_{1}}, \cdots,-\theta_{p_{n}}\right\}\right)\left\langle\left\langle-\theta_{p_{1}}, \cdots,-\theta_{p_{n}}\right|,\right.
\end{aligned}
$$

with the corresponding eigenvalues being

$$
\begin{aligned}
h\left(u,\left\{\theta_{p_{1}}, \cdots, \theta_{p_{n}}\right\}\right) & =(-1)^{N} \bar{K}_{21}^{-}(u) d(u) d(-u-\eta) \prod_{j=1}^{n} \frac{\left(u+\theta_{p_{j}}\right)\left(u-\theta_{p_{j}}+\eta\right)}{\left(u-\theta_{p_{j}}\right)\left(u+\theta_{p_{j}}+\eta\right)}, \\
h^{\prime}\left(u,\left\{-\theta_{p_{1}}, \cdots,-\theta_{p_{n}}\right\}\right) & =(-1)^{N} \bar{K}_{21}^{-}(u) a(u) a(-u-\eta) \prod_{j=1}^{n} \frac{\left(u-\theta_{p_{j}}\right)\left(u+\theta_{p_{j}}+\eta\right)}{\left(u+\theta_{p_{j}}\right)\left(u-\theta_{p_{j}}+\eta\right)} .
\end{aligned}
$$

\footnotetext{
${ }^{7}$ Similar basis was used in [21], where the open $X X Z$ spin chain was studied.
} 
For generic inhomogeneous parameters $\left\{\theta_{j}\right\}$, the above relations imply that the left states and right states satisfy the following relations

$$
\left\langle\left\langle-\theta_{q_{1}}, \cdots,-\theta_{q_{m}} \mid \theta_{p_{1}}, \cdots, \theta_{p_{n}}\right\rangle\right\rangle=f_{n}^{(o)}\left(\theta_{p_{1}}, \cdots, \theta_{p_{n}}\right) \delta_{m+n, N} \delta_{\left\{q_{1}, \cdots, q_{m}\right\} ;\left\{p_{1}, \cdots, p_{n}\right\}},
$$

where $\delta_{\left\{q_{1}, \cdots, q_{m}\right\} ;\left\{p_{1}, \cdots, p_{n}\right\}}$ is defined as

$$
\delta_{\left\{q_{1}, \cdots, q_{m}\right\} ;\left\{p_{1}, \cdots, p_{n}\right\}}= \begin{cases}1 & \text { if }\left\{q_{1}, \cdots, q_{m}, p_{1}, \cdots, p_{n}\right\}=\{1, \cdots, N\} \\ 0 & \text { otherwise }\end{cases}
$$

and $f_{n}^{(o)}\left(\theta_{p_{1}}, \cdots, \theta_{p_{n}}\right)$ is given by

$$
\begin{aligned}
f_{n}^{(o)}\left(\theta_{p_{1}}, \cdots, \theta_{p_{n}}\right)= & \left\langle\left\langle-\theta_{p_{n+1}}, \cdots,-\theta_{p_{N}} \mid \theta_{p_{1}}, \cdots, \theta_{p_{n}}\right\rangle\right\rangle \\
= & \prod_{j=1}^{n}(-1)^{N} \bar{K}_{21}^{-}\left(\theta_{p_{j}}\right) d\left(-\theta_{p_{j}}-\eta\right) a\left(\theta_{p_{j}}\right) \\
& \times \prod_{k=n+1}^{N}(-1)^{N} \bar{K}_{21}^{-}\left(-\theta_{p_{k}}\right) a\left(-\theta_{p_{k}}\right) d\left(\theta_{p_{k}}-\eta\right) \\
& \times \prod_{j=1}^{n} \prod_{l>j}^{n} \frac{\theta_{p_{j}}+\theta_{p_{l}}}{\theta_{p_{j}}+\theta_{p_{l}}+\eta} \prod_{j=n+1}^{N} \prod_{l>j}^{N} \frac{\theta_{p_{j}}+\theta_{p_{l}}}{\theta_{p_{j}}+\theta_{p_{l}}-\eta} \\
& \times \prod_{j=1}^{n} \prod_{l=n+1}^{N} \frac{\theta_{p_{l}}-\theta_{p_{j}}}{\theta_{p_{l}}-\theta_{p_{j}}-\eta} .
\end{aligned}
$$

The right states $\left.\left\{\left|\theta_{p_{1}}, \cdots, \theta_{p_{n}}\right\rangle\right\rangle\right\}$ given by (4.22) (or the left states $\left\{\left\langle\left\langle-\theta_{p_{1}}, \cdots,-\theta_{p_{n}}\right\}\right.\right.$ given by (4.23) ) form a right (or left) basis of the Hilbert space. Therefore, any right (or left) state can be decomposed as a unique linear combination of the basis.

\subsection{Retrieving the Bethe states}

Let $\left\langle\langle\Psi|\right.$ be a common eigenstate of the transfer matrix $t^{(o)}(u)$, namely,

$$
\left\langle\langle\Psi| t^{(o)}(u)=\left\langle\langle\Psi| \Lambda^{(o)}(u)\right.\right.
$$

where the eigenvalue $\Lambda^{(o)}(u)$ is given by (4.9) . Following the method used in [14], we introduce

$$
\bar{F}_{n}\left(\theta_{p_{1}}, \cdots, \theta_{p_{n}}\right)=\left\langle\left\langle\Psi \mid \theta_{p_{1}}, \cdots, \theta_{p_{n}}\right\rangle\right\rangle, n=0, \cdots, N, \quad 1 \leq p_{1}<p_{2}<\cdots<p_{n} \leq N
$$

All these quantities uniquely determine the eigenstate. Let us consider the quantity of $\left\langle\left\langle\Psi\left|t\left(\theta_{p_{n+1}}\right)\right| \theta_{p_{1}}, \cdots, \theta_{p_{n}}\right\rangle\right\rangle$. After a tedious calculation, we obtain the following recursive 
relations

$$
\Lambda^{(o)}\left(\theta_{p_{n+1}}\right) \bar{F}_{n}\left(\theta_{p_{1}}, \cdots, \theta_{p_{n}}\right)=\frac{\left(2 \theta_{p_{n+1}}+\eta\right) \bar{K}_{11}^{+}\left(\theta_{p_{n+1}}\right)+\eta \bar{K}_{22}^{+}\left(\theta_{p_{n+1}}\right)}{2 \theta_{p_{n+1}}+\eta} \bar{F}_{n+1}\left(\theta_{p_{1}}, \cdots, \theta_{p_{n+1}}\right) .
$$

The above relation allows us to determine $\left\{\bar{F}_{n}\left(\theta_{p_{1}}, \cdots, \theta_{p_{n}}\right)\right\}$ as

$$
\bar{F}_{n}\left(\theta_{p_{1}}, \cdots, \theta_{p_{n}}\right)=\left\{\prod_{j=1}^{n} \frac{\left(2 \theta_{p_{j}}+\eta\right) \Lambda^{(o)}\left(\theta_{p_{j}}\right)}{\left(2 \theta_{p_{j}}+\eta\right) \bar{K}_{11}^{+}\left(\theta_{p_{j}}\right)+\eta \bar{K}_{22}^{+}\left(\theta_{p_{j}}\right)}\right\} \bar{F}_{0}
$$

where $\bar{F}_{0}=\left\langle\langle\Psi \mid \Omega\rangle_{\xi}\right.$ is an overall scalar factor. With (4.9) in mind, we further rewrite the above expression as follows

$$
\begin{aligned}
\bar{F}_{n}\left(\theta_{p_{1}}, \cdots, \theta_{p_{n}}\right) & =\left\langle\left\langle\Psi \mid \theta_{p_{1}}, \cdots, \theta_{p_{n}}\right\rangle\right\rangle \\
& =\left\{\prod_{j=1}^{n}(-1)^{N}\left(\theta_{p_{j}}+p\right) a\left(\theta_{p_{j}}\right) d\left(-\theta_{p_{j}}-\eta\right) \frac{Q\left(\theta_{p_{j}}-\eta\right)}{Q\left(\theta_{p_{j}}\right)}\right\} \bar{F}_{0}, \\
n & =0, \cdots, N, \quad 1 \leq p_{1}<p_{2}<\cdots<p_{n} \leq N .
\end{aligned}
$$

The definitions (3.7) and (4.3) of the state $\langle 0|$ and the operators $A(u), B(u), C(u)$ and $D(u)$ allow us to derive the following relations

$$
\langle 0| A(u)=a(u)\langle 0|, \quad\langle 0| D(u)=d(u)\langle 0|, \quad\langle 0| B(u)=0, \quad\langle 0| C(u) \neq 0,
$$

where the functions $a(u)$ and $d(u)$ are given by (4.8). The expression (4.19) of the double-row monodromy matrix $\mathbb{T}(u)$ leads to the actions (see $(\overline{B .10})-(\bar{B} .13)$ ) of the matrix elements of $\mathbb{T}(u)$ on the state $\langle 0|$. The relations (4.20) between the matrix elements of $\overline{\mathbb{T}}(u)$ and those of $\mathbb{T}(u)$ allow us to derive the following expressions of $\left.\left\{\left\langle 0 \mid \theta_{p_{1}}, \cdots, \theta_{p_{n}}\right\rangle\right\rangle\right\}$

$$
\begin{aligned}
\left.\left\langle 0 \mid \theta_{p_{1}}, \cdots, \theta_{p_{n}}\right\rangle\right\rangle & =\left\{\prod_{j=1}^{n}(-1)^{N}\left(\theta_{p_{j}}+p\right) a\left(\theta_{p_{j}}\right) d\left(-\theta_{p_{j}}-\eta\right)\right\}\langle 0 \mid \Omega\rangle_{\xi}, \\
n & =0, \cdots, N, \quad 1 \leq p_{1}<p_{2}<\cdots<p_{n} \leq N .
\end{aligned}
$$

The proof of the above expression is relegated to Appendix B. With the help of (4.15) and (4.18), it is easy to check that for a generic nonzero $\xi$ the overall constant $\langle 0 \mid \Omega\rangle_{\xi}$ does not vanish. For each solution of the BAEs (4.11), let us introduce the following left Bethe states

$$
{ }_{B}\left\langle\lambda_{1}, \cdots, \lambda_{N}\right|=\langle 0|\left\{\prod_{j=1}^{N} \frac{\overline{\mathcal{C}}\left(\lambda_{j}\right)}{(-1)^{N} \bar{K}_{21}^{-}\left(\lambda_{j}\right) d\left(\lambda_{j}\right) d\left(-\lambda_{j}-\eta\right)}\right\} .
$$


The relations (4.24), (4.26) and (4.35) imply that

$$
\begin{aligned}
\left.{ }_{B}\left\langle\lambda_{1}, \cdots, \lambda_{N} \mid \theta_{p_{1}}, \cdots, \theta_{p_{n}}\right\rangle\right\rangle & =\left\{\prod_{j=1}^{n}(-1)^{N}\left(\theta_{p_{j}}+p\right) a\left(\theta_{p_{j}}\right) d\left(-\theta_{p_{j}}-\eta\right) \frac{Q\left(\theta_{p_{j}}-\eta\right)}{Q\left(\theta_{p_{j}}\right)}\right\}\langle 0 \mid \Omega\rangle_{\xi}, \\
n & =0, \cdots, N, \quad 1 \leq p_{1}<p_{2}<\cdots<p_{n} \leq N .
\end{aligned}
$$

Comparing the above expression with (4.33), we conclude that the Bethe state ${ }_{B}\left\langle\lambda_{1}, \cdots, \lambda_{N}\right|$ given by (4.36) is an eigenstate of the transfer matrix $t^{(o)}(u)$ with the corresponding eigenvalue (4.9), provided that the parameters $\left\{\lambda_{j}\right\}$ satisfy the BAEs (4.11).

With the same procedure, we can construct the right Bethe state (up to an irrelevant scalar factor) as

$$
\left|\lambda_{1}, \cdots, \lambda_{N}\right\rangle_{B}=\prod_{j=1}^{N} \overline{\mathcal{B}}\left(\lambda_{j}\right)|0\rangle,
$$

which is exactly the eigenstate conjectured in [19].

It follows from their definitions that the two "reference" states $|0\rangle$ and $\langle 0|$ are independent of the inhomogeneous parameters $\left\{\theta_{j}\right\}$ and therefore the Bethe state ${ }_{B}\left\langle\lambda_{1}, \cdots, \lambda_{N}\right|$ (or $\left.\left|\bar{\lambda}_{1}, \cdots, \bar{\lambda}_{N}\right\rangle_{B}\right)$ has a well-defined homogeneous limit.

\section{Conclusions}

In conclusion, a systematic method to retrieve the Bethe-type eigenstates of quantum integrable models is proposed. As examples, the eigenstates of the XXZ spin- $\frac{1}{2}$ torus model and the open Heisenberg chain with generic boundary fields are derived based on the ODBA solutions.

It should be remarked that constructing the Bethe-type eigenstates of generic quantum integrable models without obvious reference state had challenged for many years. The present method provides an efficient way to retrieve reference states based on inhomogeneous $T-Q$ relation that can be derived via ODBA. Naturally, this method can be generalized to other integrable models without obvious reference state. For a given monodromy matrix, some mutually commutative elements $T^{i j}(u)$, i.e., $\left[T^{i j}(u), T^{i j}(v)\right]$, exist. The eigenstates of $T^{i j}(u)$ thus form an orthogonal and complete basis (Sklyanin's SoV basis) of the Hilbert space. This basis together with the $T-Q$ relation constructed from ODBA and the commutation

relations among the elements of the monodromy matrix, allow us to retrieve the Bethe-type eigenstates of the transfer matrix step by step even without using a trial initial state. 


\section{Acknowledgments}

We would like thank R.I. Nepomechie for his valuable discussions. This work was financial supported by NSFC under grant Nos. 11375141, 11374334, 11434013, 11425522, the 973 project under grant No.2011CB921700, BCMIIS and the Strategic Priority Research Program of CAS.

\section{Appendix A: Proof of (3.23) satisfying (3.22)}

Let us introduce the following inner product

$$
\left\langle\theta_{1}, \cdots, \theta_{n}\left|\prod_{k=1}^{m} \bar{B}\left(u_{k}\right)\right| 0\right\rangle=\delta_{n, m} g_{n}\left(\left\{\theta_{j}\right\} \mid\left\{u_{\alpha}\right\}\right), \quad g_{0}=\langle 0 \mid 0\rangle=1 .
$$

The relations (3.4)-(3.6) allow us to derive some recursive relations for the function $g_{n}\left(\left\{\theta_{j}\right\} \mid\right.$ $\left.\left\{u_{\alpha}\right\}\right):$

$$
\begin{aligned}
g_{n}\left(\left\{\theta_{j}\right\} \mid\left\{u_{\alpha}\right\}\right)= & \sum_{l=1}^{n} \frac{\sinh \eta \bar{d}\left(u_{1}\right) \bar{a}\left(\theta_{l}\right)}{\sinh \left(u_{1}-\theta_{l}\right)}\left\{\prod_{j \neq l}^{n} \frac{\sinh \left(u_{1}-\theta_{j}+\eta\right)}{\sinh \left(u_{1}-\theta_{j}\right)}\right. \\
& \left.\times \frac{\sinh \left(\theta_{l}-\theta_{j}-\eta\right)}{\sinh \left(\theta_{l}-\theta_{j}\right)}\right\} g_{n-1}\left(\left\{\theta_{j}\right\}_{j \neq l} \mid\left\{u_{\alpha}\right\}_{\alpha \neq 1}\right) .
\end{aligned}
$$

Moreover, let us introduce the following quantity

$$
\bar{g}_{n}\left(\left\{\theta_{p_{j}}\right\}\right)=[n]_{q} !\left\langle\theta_{p_{1}}, \cdots, \theta_{p_{n}} \mid \Omega ;\left\{\theta_{j}\right\}\right\rangle=\left\langle\theta_{p_{1}}, \cdots, \theta_{p_{n}}\left|\left(\tilde{B}^{-}\right)^{n}\right| 0\right\rangle .
$$

The definitions (A.1) and (3.26) imply that one can calculate the function $\bar{g}_{n}\left(\left\{\theta_{j}\right\}\right)$ by the following limit

$$
\bar{g}_{n}\left(\left\{\theta_{j}\right\}\right)=\lim _{\left\{u_{l} \rightarrow+\infty\right\}}\left\{\left[\prod_{l=1}^{n}\left(2 \sinh \eta e^{-u_{l}}\right)^{N-1} e^{\sum_{k=1}^{N} \theta_{k}}\right] g_{n}\left(\left\{\theta_{j}\right\} \mid\left\{u_{l}\right\}\right)\right\} .
$$

Keeping the recursive relations (A.2) in mind, we can derive the following recursive relations

$$
\begin{aligned}
\bar{g}_{n}\left(\left\{\theta_{j}\right\}\right) & =\sum_{l=1}^{n} e^{(n-1) \eta} a\left(\theta_{l}\right) e^{\theta_{l}} \prod_{j \neq l}^{n} \frac{\sinh \left(\theta_{l}-\theta_{j}-\eta\right)}{\sinh \left(\theta_{l}-\theta_{j}\right)} \bar{g}_{n-1}\left(\left\{\theta_{j}\right\}_{j \neq l}\right), \\
n & =1, \cdots, N
\end{aligned}
$$

with the initial condition of $\bar{g}_{0}=1$. The above recursive relations uniquely determine the functions $\left\{\bar{g}_{n}\left(\left\{\theta_{j}\right\}\right) \mid n=0, \cdots, N\right\}$ :

$$
\bar{g}_{n}\left(\left\{\theta_{j}\right\}\right)=\left\{\prod_{l=1}^{n} a\left(\theta_{l}\right) e^{\theta_{l}}\right\}[n]_{q} !, \quad n=0, \cdots, N .
$$


Note that the following identities were used in deriving the above equations

$$
\sum_{l=1}^{n} e^{(n-1) \eta} \prod_{j \neq l}^{n} \frac{\sinh \left(\theta_{l}-\theta_{j}-\eta\right)}{\sinh \left(\theta_{l}-\theta_{j}\right)}=1+e^{2 \eta}+\cdots+e^{2(n-1) \eta}=[n]_{q} .
$$

Substituting (A.5) into (A.3), we find that the state $\left|\Omega ;\left\{\theta_{j}\right\}\right\rangle$ given by (3.23) indeed satisfies the relations (3.22).

\section{Appendix B: Proof of (4.35)}

The gauged double-row monodromy matrix $\overline{\mathbb{T}}(u)$ given by (4.20) satisfies the reflection algebra [3]

$$
R_{12}(u-v) \overline{\mathbb{T}}_{1}(u) R_{21}(u+v) \overline{\mathbb{T}}_{2}(v)=\overline{\mathbb{T}}_{2}(v) R_{12}(u+v) \overline{\mathbb{T}}_{1}(u) R_{21}(u-v),
$$

which leads to the following relevant relations

$$
\begin{aligned}
\overline{\mathcal{C}}(u) \overline{\mathcal{A}}(v)= & \frac{(u+v)(u-v+\eta)}{(u-v)(u+v+\eta)} \overline{\mathcal{A}}(v) \overline{\mathcal{C}}(u)-\frac{\eta}{u+v+\eta} \overline{\mathcal{D}}(u) \overline{\mathcal{C}}(v) \\
& -\frac{(u+v) \eta}{(u-v)(u+v+\eta)} \overline{\mathcal{A}}(u) \overline{\mathcal{C}}(v), \\
\overline{\mathcal{D}}(v) \overline{\mathcal{C}}(u)= & \frac{(u+v)(u-v+\eta)}{(u-v)(u+v+\eta)} \overline{\mathcal{C}}(u) \overline{\mathcal{D}}(v)-\frac{\eta}{u+v+\eta} \overline{\mathcal{C}}(v) \overline{\mathcal{A}}(u) \\
\overline{\mathcal{A}}(u) \overline{\mathcal{A}}(v)= & -\frac{(u+v) \eta}{(u-v)(u+v+\eta)} \overline{\mathcal{\mathcal { C }}}(v) \overline{\mathcal{A}}(u)+\frac{\eta}{u+v+\eta} \overline{\mathcal{B}}(u), \\
\overline{\mathcal{D}}(u) \overline{\mathcal{D}}(v)= & \overline{\mathcal{D}}(v) \overline{\mathcal{D}}(u)+\frac{\eta}{u+v+\eta} \overline{\mathcal{C}}(v) \overline{\mathcal{B}}(u)-\frac{\eta}{u+v+\eta} \overline{\mathcal{B}}(u) \overline{\mathcal{C}}(v), \overline{\mathcal{C}}(u) \overline{\mathcal{B}}(v), \\
\overline{\mathcal{D}}(u) \overline{\mathcal{A}}(v)= & \overline{\mathcal{A}}(v) \overline{\mathcal{D}}(u)-\frac{(u+v+2 \eta) \eta}{(u-v)(u+v+\eta)} \overline{\mathcal{B}}(u) \overline{\mathcal{C}}(v) \\
& +\frac{(u+v+2 \eta) \eta}{(u-v)(u+v+\eta)} \overline{\mathcal{B}}(v) \overline{\mathcal{C}}(u) .
\end{aligned}
$$

Equations (4.13) and (4.20) imply that the following relations hold:

$$
\begin{aligned}
\overline{\mathcal{A}}(u)= & \frac{1}{2 \xi \sqrt{1+\xi^{2}}}\left\{\xi\left(1+\sqrt{1+\xi^{2}}\right) \mathcal{A}(u)+\xi^{2} \mathcal{C}(u)\right. \\
& \left.+\xi^{2} \mathcal{B}(u)-\xi\left(1-\sqrt{1+\xi^{2}}\right) \mathcal{D}(u)\right\} \\
\overline{\mathcal{C}}(u)= & \frac{1}{2 \xi \sqrt{1+\xi^{2}}}\left\{\xi\left(1+\sqrt{1+\xi^{2}}\right) \mathcal{A}(u)-\left(1+\sqrt{1+\xi^{2}}\right)^{2} \mathcal{C}(u)\right.
\end{aligned}
$$




$$
\begin{aligned}
& \left.+\xi^{2} \mathcal{B}(u)-\xi\left(1+\sqrt{1+\xi^{2}}\right) \mathcal{D}(u)\right\}, \\
\overline{\mathcal{D}}(u)= & \frac{1}{2 \xi \sqrt{1+\xi^{2}}}\left\{\xi\left(\sqrt{1+\xi^{2}}-1\right) \mathcal{A}(u)-\xi^{2} \mathcal{C}(u)\right. \\
& \left.-\xi^{2} \mathcal{B}(u)+\xi\left(1+\sqrt{1+\xi^{2}}\right) \mathcal{D}(u)\right\} .
\end{aligned}
$$

We note that

$$
\begin{aligned}
\langle 0| \mathcal{A}(u)= & (-1)^{N} K_{11}^{-}(u) a(u) d(-u-\eta)\langle 0|, \\
\langle 0| \mathcal{D}(u)= & (-1)^{N} \frac{\eta}{2 u+\eta} K_{11}^{-}(u) a(u) d(-u-\eta)\langle 0| \\
& +(-1)^{N} \frac{(2 u+\eta) K_{22}^{-}(u)-\eta K_{11}^{-}(u)}{2 u+\eta} d(u) a(-u-\eta)\langle 0|, \\
\langle 0| \mathcal{B}(u)= & 0, \\
\langle 0| \mathcal{C}(u)= & (-1)^{N} \frac{2 u}{2 u+\eta} K_{11}^{-}(u) d(-u-\eta)\langle 0| C(u) \\
& +(-1)^{N} \frac{\eta K_{11}^{-}(u)-(2 u+\eta) K_{22}^{-}(u)}{2 u+\eta} d(u)\langle 0| C(-u-\eta) .
\end{aligned}
$$

Let us consider the quantity $\left.\left\langle 0\left|\overline{\mathcal{C}}\left(\theta_{p_{n+1}}\right)\right| \theta_{p_{1}}, \cdots, \theta_{p_{n}}\right\rangle\right\rangle$. Acting $\overline{\mathcal{C}}\left(\theta_{p_{n+1}}\right)$ to the right, from the relations (4.24) and (4.26), we know that $\left.\left\langle 0\left|\overline{\mathcal{C}}\left(\theta_{p_{n+1}}\right)\right| \theta_{p_{1}}, \cdots, \theta_{p_{n}}\right\rangle\right\rangle$ vanishes. On the other hand, acting it to the left and using $(\overline{B .10})-(\overline{B .13})$ and the relation $(\overline{B .8})$, we obtain

$$
\left.\left.\left\langle 0\left|C\left(\theta_{p_{n+1}}\right)\right| \theta_{p_{1}}, \cdots, \theta_{p_{n}}\right\rangle\right\rangle=\frac{\xi}{1+\sqrt{1+\xi^{2}}} a\left(\theta_{p_{n+1}}\right)\left\langle 0 \mid \theta_{p_{1}}, \cdots, \theta_{p_{n}}\right\rangle\right\rangle, \quad j=1, \cdots, N .
$$

From the definition (4.22) we have

$$
\left.\left.\left\langle 0 \mid \theta_{p_{1}}, \cdots, \theta_{p_{n+1}}\right\rangle\right\rangle=\left\langle 0\left|\overline{\mathcal{A}}\left(\theta_{p_{n+1}}\right)\right| \theta_{p_{1}}, \cdots, \theta_{p_{n}}\right\rangle\right\rangle .
$$

Acting $\overline{\mathcal{A}}\left(\theta_{p_{n+1}}\right)$ to the left and using (B.10) $-(\underline{\mathrm{B} .13})$ and the relation (B.7), we have

$$
\begin{aligned}
\left.\left\langle 0 \mid \theta_{p_{1}}, \cdots, \theta_{p_{n+1}}\right\rangle\right\rangle= & \frac{(-1)^{N}}{2 \sqrt{1+\xi^{2}}}\left\{\left(1+\sqrt{1+\xi^{2}}\right) K_{11}^{-}\left(\theta_{p_{n+1}}\right) a\left(\theta_{p_{n+1}}\right) d\left(-\theta_{p_{n+1}}-\eta\right)\left\langle 0 \mid \theta_{p_{1}}, \cdots, \theta_{p_{n}}\right\rangle\right\rangle \\
& \left.-\left(1-\sqrt{1+\xi^{2}}\right) \frac{\eta}{2 \theta_{p_{n+1}}+\eta} K_{11}^{-}\left(\theta_{p_{n+1}}\right) a\left(\theta_{p_{n+1}}\right) d\left(-\theta_{p_{n+1}}-\eta\right)\left\langle 0 \mid \theta_{p_{1}}, \cdots, \theta_{p_{n}}\right\rangle\right\rangle \\
& \left.\left.+\xi \frac{2 \theta_{p_{n+1}}}{2 \theta_{p_{n+1}}+\eta} K_{11}^{-}\left(\theta_{p_{n+1}}\right) d\left(-\theta_{p_{n+1}}-\eta\right)\left\langle 0\left|C\left(\theta_{p_{n+1}}\right)\right| \theta_{p_{1}}, \cdots, \theta_{p_{n}}\right\rangle\right\rangle\right\} \\
= & \left.(-1)^{N} K_{11}^{-}\left(\theta_{p_{n+1}}\right) a\left(\theta_{p_{n+1}}\right) d\left(-\theta_{p_{n+1}}-\eta\right)\left\langle 0 \mid \theta_{p_{1}}, \cdots, \theta_{p_{n}}\right\rangle\right\rangle, \\
n= & 0, \cdots, N-1 .
\end{aligned}
$$




\section{References}

[1] E. K. Sklyanin and L. D. Faddeev, Sov. Phys. Dokl. 23 (1978), 902.

[2] L. A. Takhtadzhan and L. D. Faddeev, Rush. Math. Surveys 34 (1979), 11.

[3] E. K. Sklyanin, J. Phys. A 21 (1988), 2375.

[4] V. E. Korepin, N. M. Bogoliubov and A. G. Izergin, Quantum Inverse Scattering Method and Correlation Function, Cambridge University Press, Cambridge, 1993.

[5] J. Cao, H.-Q. Lin, K.-J. Shi and Y. Wang, Nucl. Phys. B 663 (2003), 487.

[6] W.-L. Yang and R. Sasaki, Nucl. Phys. B 679 (2004), 495; J. Math. Phys. 45 (2004), 4301.

[7] W.-L. Yang, Y.-Z. Zhang and M. Gould, Nucl. Phys. B 698 (2004), 503.

[8] A. Doikou and P. P. Martins, J. Stat. Mech. (2006), P06004; A. Doikou, J. Stat. Mech. (2006), P05010.

[9] W. -L. Yang and Y.-Z. Zhang, JHEP 04 (2007), 044; Nucl. Phys. B 789 (2008), 591.

[10] N. Crampé and E. Ragoucy, Nucl. Phys. B 858 (2012), 502.

[11] N. Karaiskos, A. M. Grabinski and H. Frahm, J. Stat. Mech. (2013), P07009.

[12] R. A. Pimenta and A. Lima-Santos, J. Phys. A 46 (2013), 455002.

[13] S. Belliard, N. Crampé and E. Ragoucy, Lett. Math. Phys. 103 (2013), 493.

[14] J. Cao, W.-L. Yang, K. Shi and Y. Wang, Phys. Rev. Lett. 111 (2013), 137201.

[15] J. Cao, W.-L. Yang, K. Shi and Y. Wang, Nucl. Phys. B 875 (2013), 152.

[16] J. Cao, W.-L. Yang, K. Shi and Y. Wang, Nucl. Phys. B 877 (2013), 152.

[17] J. Cao, W.-L. Yang, K. Shi and Y. Wang, Nucl. Phys. B 886 (2014), 185.

[18] J. Cao, W.-L. Yang, K. Shi and Y. Wang, JHEP 04 (2014), 143.

[19] S. Belliard and N. Crampé, SIGMA 9 (2013), 072. 
[20] G. Niccoli, J. Stat. Mech. (2012), P10025.

[21] G. Niccoli, Nucl. Phys. B 870 (2013), 397; J. Phys. A 46 (2013), 075003.

[22] S. Faldella, N. Kitanine and G. Niccoli, J. Stat. Mech. (2014), P01011.

[23] C. M. Yung and M. T. Batchelor, Nucl. Phys. B 446 (1995), 461.

[24] M. T. Batchelor, R. J. Baxter, M. J. O'Rourke and C. M. Yung, J. Phys. A 28 (1995), 2759.

[25] W. Galleas, Nucl. Phys. B 790 (2008), 524.

[26] S. Niekamp, T. Wirth and H. Frahm, J. Phys. A 42 (2009), 195008.

[27] H. Frahm, J. H. Grelik, A. Seel and T. Wirth, J. Phys. A 44 (2011), 015001.

[28] J. Cao, W.-L. Yang, K. Shi and Y. Wang, arXiv:1409.5303,

[29] E. K. Sklyanin, Lect. Notes Phys. 226 (1985), 196; J. Sov. Math. 31 (1985), 3417; Prog. Theor. Phys. Suppl. 118 (1995), 35.

[30] H. J. de Vega and A. González-Ruiz, J. Phys. A 26 (1993), L519.

[31] S. Ghoshal and A. B. Zamolodchikov, Int. J. Mod. Phys. A 9 (1994), 3841.

[32] R. I. Nepomechie, J. Phys. A 46 (2013), 442002. 